\title{
MINIMAL INVARIANT FUNCTIONS OF THE SPACE-TIME WIENER PROCESS
}

BY

KAI YUEN WOO

ABSTRACT. Minimal invariant functions of the space-time Wiener process are obtained.

1. Introduction. Robbins and Siegmund [9] obtained an integral representation of any nonnegative invariant function of the space-time Wiener process as

$$
h(x, t)=\int_{-\infty}^{\infty} \exp \left(\lambda x-\frac{\lambda^{2} t}{2}\right) \mu(d \lambda)
$$

for some measure $\mu$ on $(-\infty, \infty)$. These functions are also the nonnegative $c^{\infty}$ solutions of $\partial h / \partial t+\frac{1}{2}\left(\partial^{2} h / \partial x^{2}\right)=0,(x, t) \in(-\infty, \infty) \times(0, \infty)$ such that $\lim _{t \rightarrow 0} h(x, t)=h(x, 0)$ exists as a function, cf. Lai [6], McKean [7]. However, such a representation also falls into general considerations in Martin boundary theory, cf. Meyer [8]. It is the purpose of this paper to establish by elementary methods that the functions $\exp \left(\lambda x-\lambda^{2} t / 2\right)$ are minimal invariant functions of the space-time Wiener process and later on to establish the above representation by Martin boundary theory. On the other hand, Doob, Snell and Williamson [4] constructed minimal invariant functions of random walks on the $N$-dimensional lattice. Our result will offer an example in the continuous time case.

2. Definitions and main results. We shall follow Dynkin [5] in the definition and notation of a Markov process. Let $X^{\prime}=\left(x_{t}^{1}, \zeta^{1}, \Re_{t}^{1}, P_{x^{1}}^{1}\right)$ be a Wiener process on the real line $R$ with almost all sample functions continuous. Let $X^{2}=\left(x_{t}^{2}, \zeta^{2}, \Re_{t}^{2}, P_{x^{2}}^{2}\right)$ be the process of uniform motion to the right on $[0, \infty)$ with transition function $P\left(t, x^{2}, T\right)=\chi_{T}\left(x^{2}+t\right)\left(x^{2} \in[0, \infty)\right) . X^{1}$ and $X^{2}$ are Markov processes and their joint process $X=\left(X^{1}, X^{2}\right)=\left(x_{t}, \zeta, \Re_{t}, P_{x}\right)$ is a Markov process on $R \times[0, \infty)$ with transition function $P(t, x, A)$ generated

Presented to the Society, April 14, 1973 under the title $A$ characterization of $P_{t}$-invariant functions of the space-time Wiener process; received by the editors February 15, 1975 and, in revised form, February 7, 1976.

AMS (MOS) subject classifications (1970). Primary 60J50; Secondary $60 \mathrm{~J} 65$.

Key words and phrases. Space-time Wiener process, minimal invariant functions. 
by assignments on measurable rectangles of $\mathscr{B}(\Re) \times \mathscr{B}([0, \infty))$ given by

$$
P\left(t,\left(x^{1}, x^{2}\right), \Gamma \times T\right)=\chi_{T}\left(x^{2}+t\right) \int_{\Gamma} \frac{1}{\sqrt{2 \pi t}} \exp \left(-\frac{\left(y-x^{1}\right)^{2}}{2 t}\right) d y
$$

where we write $\left(x^{1}, x^{2}\right)$ for $x$. This joint process $X$ we shall call the space-time Wiener process. It is a Markov process with almost all sample functions continuous. For convenience we denote the state space $(R \times[0, \infty), \mathscr{B}(\Re)$ $\times \Re([0, \infty)))$ of $X$ by $(E, \mathscr{B})$ and any point in $E$ by $(x, u)$. A nonnegative measurable function $h$ on $(E, \Re)$ is said to be invariant (for $X)$ if

$$
P_{t} h=h \text { for all } t \geqslant 0
$$

where

$$
P_{t} h(x, u)=\int_{E} P(t,(x, u), d(y, v)) h(y, v) \quad((x, u) \in E) .
$$

An invariant function $h$ is said to be minimal if for any other invariant function $g$ such that $0 \leqslant g \leqslant h$ we have $g=c h$ for some constant $c$. The main theorems obtained in this paper are the following:

THEOREM 1. Let $h$ be a nonnegative measurable function on $(E, B)$ which satisfies $h(0,0)=1$. Then $h$ is a minimal invariant function if and only if the following two conditions are satisfied:

(a) $P_{t} h(0,0)=1$ for all $t \geqslant 0$,

(b) $h[(x, u)+(y, v)]=h(x, u) h(y, v)$ for all $(x, u),(y, v) \in E$.

THEOREM 2. The minimal invariant functions $h$ for the space-time Wiener process which satisfy $h(0,0)=1$ are $\exp \left(\alpha x-\alpha^{2} u / 2\right)((x, u) \in E)$ where $\alpha$ runs through $(-\infty, \infty)$.

The rest of the paper will be devoted to proofs of the above theorems.

3. Proof of Theorem 1. We first prove the necessity part of Theorem 1 . We shall denote by $p(t, x, \Gamma)$ the transition function of the Wiener process on the real line $R$.

LEMMA 1. If $h$ is a nonnegative measurable function on $(E, \mathscr{B})$, then for any $t \geqslant 0$,

(a) $\quad P_{t} h(x, u)=\int_{R} p(t, x, d y) h(y, u+t) \quad((x, u) \in E)$

and

(b)

$$
\begin{array}{r}
P_{t} h(x+y, u+v)=\int_{E} P(t,(x, u), d(z, w)) h[(z, w)+(y, v)] \\
((x, u),(y, v) \in E) .
\end{array}
$$


ProOF.

(a)

$$
\begin{aligned}
P_{t} h(x, u) & =\int_{E} P(t,(x, u), d(y, v)) h(y, v) \\
& =\int_{R \times\{u+t\}} P(t,(x, u), d(y, v)) h(y, v) \\
& =\int_{R} p(t, x, d y) h(y, u+t) .
\end{aligned}
$$

(b) $\quad P_{t} h(x+y, u+v)=\int_{E} P(t,(x+y, u+v), d(z, w)) h(z, w)$

$$
\begin{aligned}
& =\int_{R} p(t, x+y, d z) h(z, u+v+t) \\
& =\int_{R} p(t, x, d z) h(y+z, u+v+t) \\
& =\int_{E} P(t,(x, u), d(z, w)) h(y+z, v+w) \\
& =\int_{E} P(t,(x, u), d(z, w)) h[(z, w)+(y, v)] . \quad \text { Q.E.D. }
\end{aligned}
$$

LEMMA 2. If $h$ is an invariant function, $h(0,0)=1$, then $h$ is positive everywhere and for each $u>0, h(x, u)$ is finite and is a continuous function in $x$.

Proof. (a) Suppose $h\left(x_{0}, u_{0}\right)=0$ for some $\left(x_{0}, u_{0}\right) \in E$. By the invariant property we have for any $t>0, h\left(y, u_{0}+t\right)=0$ for almost every $y$ on $R$, which by the invariant property again implies $h(0,0)=0$, a contradiction.

(b) Let $u>0$ be arbitrarily fixed. For any $t>0$,

$$
\begin{aligned}
& h(x, u)=\int_{R} p(t, x, d y) h(y, u+t) \\
&=\int_{R} \frac{1}{\sqrt{2 \pi t}} \exp \left(-\frac{(y-x)^{2}}{2 t}\right) h(y, u+t) d y \\
&=\int_{R} \frac{1}{\sqrt{2 \pi t}} \exp \left(-\frac{(y-x)^{2}}{2 t}\right) \sqrt{2 \pi(u+t)} \exp \left(\frac{y^{2}}{2(u+t)}\right) \\
&=\int_{R} \sqrt{\frac{u+t}{t}} \exp \left(-\frac{1}{\sqrt{2 \pi(u+t)}} \exp \left(-\frac{y^{2}}{2(u+t)}\right) h(y, u+t) d y\right. \\
& \times \frac{1}{\sqrt{2 \pi(u+t)}} \exp \left(-\frac{y^{2}}{2(u+t)}\right) h(y, u+t) d y .
\end{aligned}
$$


Since

$$
\int_{R} \frac{1}{\sqrt{2 \pi(u+t)}} \exp \left(-\frac{y^{2}}{2(u+t)}\right) h(y, u+t) d y=h(0,0)=1
$$

and $u>0, h(x, u)$ is finite for all $x \in R$, and by the dominated convergence theorem we have $\lim _{x \rightarrow x_{0}} h(x, u)=h\left(x_{0}, u\right)$. Q.E.D.

LEMMA 3. Let $h$ be an invariant function, $h(0,0)=1$. Let $\left(y_{0}, v_{0}\right) \in E, v_{0}$ $>0$, be arbitrarily fixed. Let $U_{n}=\left\{(y, v) \in E:\left|y-y_{0}\right|<1 / n\right\}$ and $\mu_{v_{0}}$ be the measure on $(E, \mathscr{B})$ such that

$$
\mu_{v_{0}}(A)=\int_{A} P\left(v_{0},(0,0), d(y, v)\right) h(y, v) \quad(A \in \mathfrak{B}) .
$$

Then

(a) $0<\mu_{v_{0}}\left(U_{n}\right)<\infty(n=1,2, \ldots)$,

(b) the functions

$$
h_{n}(x, u)=\int_{U_{n}} \frac{h[(x, u)+(y, v)]}{h(y, v)} \mu_{v_{0}}(d(y, v))
$$

are invariant, and

(c) $0 \leqslant h_{n} \leqslant h$.

ProOF. (a)

$$
\begin{aligned}
\mu_{v_{0}}\left(U_{n}\right) & =\int_{U_{n}} P\left(v_{0},(0,0), d(y, v)\right) h(y, v) \\
& =\int_{\left(y_{0}-1 / n, y_{0}+1 / n\right)} p\left(v_{0}, 0, d y\right) h\left(y, v_{0}\right) \\
& =\int_{\left(y_{0}-1 / n, y_{0}+1 / n\right)} \frac{1}{\sqrt{2 \pi v_{0}}} \exp \left(-\frac{y^{2}}{2 v_{0}}\right) h\left(y, v_{0}\right) d y .
\end{aligned}
$$

Since by Lemma $2, h\left(y, v_{0}\right)$ is positive and continuous in $y$, the result follows.

(b) and (c) follow from Lemma 1(b). Q.E.D.

To prove the necessity part of Theorem 1 we assume that $h$ is a minimal invariant function on $(E, \mathscr{B})$ with $h(0,0)=1$. We shall show that (a) and (b) of Theorem 1 are satisfied.

For (a), $P_{t} h(0,0)=h(0,0)=1$ for all $t \geqslant 0$.

For (b), let $\left(y_{0}, v_{0}\right) \in E, v_{0}>0$, be arbitrarily fixed and let $U_{n}, \mu_{v_{0}}$ and $h_{n}(x, u)$ be as defined in Lemma 3. By the same lemma the functions $h_{n}$ are invariant and satisfy $0 \leqslant h_{n} \leqslant h$. By the minimality of $h$ we have $h_{n}=c_{n} h$ for some constants $c_{n} \cdot c_{n}=h_{n}(0,0)=\mu_{v_{0}}\left(U_{n}\right)$ because $h(0,0)=1$. Thus 


$$
\begin{aligned}
h(x, u) & =\frac{1}{\mu_{v_{0}}\left(U_{n}\right)} \int_{U_{n}} \frac{h[(x, u)+(y, v)]}{h(y, v)} \mu_{v_{0}}(d(y, v)) \\
& =\frac{1}{\mu_{v_{0}}\left(U_{n}\right)} \int_{U_{n}} \frac{h\left[(x, u)+\left(y, v_{0}\right)\right]}{h\left(y, v_{0}\right)} \mu_{v_{0}}(d(y, v)) .
\end{aligned}
$$

The continuity of $h\left(y, v_{0}\right)$ in $y$ implies that as $n \rightarrow \infty$, the right-hand side of the above equation tends to $h\left[(x, u)+\left(y_{0}, v_{0}\right)\right] / h\left(y_{0}, v_{0}\right)$. The left-hand side being independent of $n$, we have

$$
h(x, u)=h\left[(x, u)+\left(y_{0}, v_{0}\right)\right] / h\left(y_{0}, v_{0}\right) .
$$

Since $\left(y_{0}, v_{0}\right) \in E$ is arbitrary, subject only to the condition $v_{0}>0$,

$$
h[(x, u)+(y, v)]=h(x, u) h(y, v)
$$

holds for all $(x, u),(y, v) \in E$ such that not both $u$ and $v$ are zero. If $u=v=0$, then for any $t>0$,

$$
\begin{aligned}
h((x, 0)+(y, 0)) & =h(x+y, 0)=P_{t} h(x+y, 0) \\
& =\int_{E} P(t,(x, 0), d(z, w)) h[(z, w)+(y, 0)] \\
& =\int_{R} p(t, x, d z) h[(z, t)+(y, 0)]=\int_{R} p(t, x, d z) h(z, t) h(y, 0) \\
& =\left[\int_{E} P(t,(x, 0), d(z, w)) h(z, w)\right] h(y, 0) \\
& =h(x, 0) h(y, 0) .
\end{aligned}
$$

Thus the necessity part of Theorem 1 is proved.

Next we prove the sufficiency part of Theorem 1 . Throughout the rest of this section we shall assume that $h$ is a nonnegative measurable function on $(E, \mathscr{B})$ such that $h(0,0)=1$ and conditions (a) and (b) of Theorem 1 are satisfied. We shall show that $h$ is a minimal invariant function.

LEMMA 4. $h$ is an invariant function, finite and positive everywhere on $E$.

Proof. (a) For any $t \geqslant 0$, by Lemma 1 ,

$$
\begin{aligned}
P_{t} h(x, u) & =P_{t} h(0+x, 0+u) \\
& =\int_{E} P(t,(0,0), d(z, w)) h[(z, w)+(x, u)] \\
& =\int_{E} P(t,(0,0), d(z, w)) h(z, w) h(x, u) \\
& =\left[P_{t} h(0,0)\right] h(x, u)=h(x, u) .
\end{aligned}
$$


(b) From Lemma 2 and above we see that $h(x, u)$ is finite and positive for all $(x, u) \in E$ such that $u>0$. If $u>0$, then for any $v>0$,

$$
h(x, 0) h(0, v)=h(x+0,0+v)=h(x, v)
$$

for any $x$. Since $h(x, v)$ and $h(0, v)$ are finite and positive, $h(x, 0)$ is finite and positive also. Q.E.D.

LEMma 5. The function $q(t, x, \Gamma)(t \geqslant 0, x \in R, \Gamma \in \mathscr{B}(R))$, defined as

$$
q(t, x, \Gamma)=\frac{1}{h(x, 0)} \int_{\Gamma} p(t, x, d y) h(y, t),
$$

is a transition function on $(R, \mathscr{B}(R))$ with $q(t, x, R)=1$.

Proof. $q(t, x, \Gamma)$ is well defined because of Lemma 4. For each $t \geqslant 0$, it is easy to see that $q(t, x, \cdot)$ is a measure on $\mathscr{B}(R)$ for each $x \in R$ and $q(t, \cdot, \Gamma)$ is a $\mathfrak{B}(R)$-measurable function for each $\Gamma \in \mathscr{B}(R), q(t, x, R)=1$ because

$$
\begin{aligned}
q(t, x, R) & =\frac{1}{h(x, 0)} \int_{R} p(t, x, d y) h(y, t) \\
& =[1 / h(x, 0)]\left[P_{t} h(x, 0)\right]=h(x, 0) / h(x, 0)=1 .
\end{aligned}
$$

Finally,

$$
\begin{aligned}
\int_{R} q(s, x, d y) q(t, y, \Gamma) \\
\quad=\int_{R} \frac{1}{h(x, 0)} p(s, x, d y) h(y, s) \frac{1}{h(y, 0)} \int_{\Gamma} p(t, y, d z) h(z, t) \\
=\frac{h(0, s)}{h(x, 0)} \int_{R} p(s, x, d y) \int_{R} p(t, y, d z) \chi_{\Gamma}(z) h(z, t) \\
=\frac{h(0, s)}{h(x, 0)} \int_{R} p(s+t, x, d z) \chi_{\Gamma}(z) h(z, t) \\
=\frac{1}{h(x, 0)} \int_{\Gamma} p(s+t, x, d z) h(z, s+t) \\
=q(s+t, x, \Gamma) . \quad \text { Q.E.D. }
\end{aligned}
$$

By a general theorem (see [5]) there exists a Markov process $Y^{1}=\left(y_{t}^{1}, \zeta^{1}\right.$, $\left.\mathfrak{N}_{t}^{1}, P_{y^{1}}^{1}\right)$ on $(R, \mathscr{B}(R))$ which has $q(t, x, \Gamma)$ as its transition function. Moreover we can regard $\zeta^{1} \equiv \infty$ because $q(t, x, R)=1$. If we consider the joint process of $Y^{1}$ with the process of uniform motion to the right we obtain a Markov process $Y=\left(y_{t}, \zeta, \Re_{t}, P_{y}\right)$ on $E=R \times[0, \infty)$ with $\zeta \equiv \infty$. Its transition function $Q(t,(x, u), A)$ is given by 


$$
Q(t,(x, u), A)=\frac{1}{h(x, u)} \int_{A} P(t,(x, u), d(y, v)) h(y, v)
$$

for every $A \in \mathscr{B}$.

LEMMA 6. If $r$ is any nonnegative measurable function on $(E, \Re)$, then for every $t \geqslant 0,(x, u) \in E$,

$\int_{E} Q(t,(x, u), d(y, v)) r(y, v)=\int_{E} Q(t,(0,0), d(y, v)) r[(x, u)+(y, v)] . \quad$ Q.E.D.

Proof. As in Lemma 1(b).

Since $Q(t,(x, u), A)$ is a transition function, we can define a nonnegative measurable function $q$ on $(E, \Re)$ to be invariant with respect to $Q$ if $Q_{t} q=q$ for all $t \geqslant 0$, where

$$
Q_{t} q(x, u)=\int_{E} Q(t,(x, u), d(y, v)) q(y, v) \quad((x, u) \in E) .
$$

For convenience we call such a function $Q_{t}$-invariant. We shall also define a minimal $Q_{t}$-invariant function to be a $Q_{t}$-invariant function $q$ such that for every $Q_{t}$-invariant function $r$ with $0 \leqslant r \leqslant q$ we have $r=c q$ for some constant $c$. We shall proceed to show that the constant function 1 is a minimal $Q_{t}$-invariant function. First we prove two lemmas.

LEMMA 7. If $r$ is a $Q_{t}$-invariant function, $r(0,0)<\infty$, then for each $u>0$, $r(x, u)$ is a continuous function in $x$.

Proof. It is clear that $r$ is $Q_{t}$-invariant if and only if $r h$ is invariant. Hence the continuity of $r(x, u)$ in $x$ for $u>0$ follows from Lemma 2. Q.E.D.

Regarding the Markov process $Y=\left(y_{t}, \zeta, \Re_{t}, P_{y}\right)$ as a stochastic process $\left\{y_{t}: t \geqslant 0\right\}$ on $(E, \mathscr{B})$ with a probability space $\left(\Omega, \mathscr{\Re}, P_{(0,0)}\right)$, we prove

LEMMA 8. The stochastic process $\left\{y_{t}: t \geqslant 0\right\}$ has stationary independent increments.

Proof. (a) The increments are stationary because for any $A \in \mathscr{B}, 0 \leqslant s$ $<t$,

$$
\begin{aligned}
P_{(0,0)}\left\{y_{t}-y_{s} \in A\right\} & \\
= & \int_{E} Q(s,(0,0), d(y, v)) \int_{E} Q(t-s,(y, v), d(z, w)) \\
& \quad \times \chi_{\{(z, w)-(y, v) \in A\}}((y, v),(z, w))
\end{aligned}
$$

which by Lemma 6 is 


$$
\begin{aligned}
& =\int_{E} Q(s,(0,0), d(y, v)) \int_{E} Q(t-s,(0,0), d(z, w)) \chi_{A}(z, w) \\
& =Q(t-s,(0,0), A) .
\end{aligned}
$$

(b) The increments are independent because for any $A_{1}, \ldots, A_{n-1} \in \mathscr{B}, 0$ $\leqslant t_{1}<t_{2}<\cdots<t_{n}$,

$$
\begin{gathered}
P_{(0,0)}\left\{y_{t_{2}}-y_{t_{1}} \in A_{1}, y_{t_{3}}-y_{t_{2}} \in A_{2}, \ldots, y_{t_{n}}-y_{t_{n-1}} \in A_{n-1}\right\} \\
=\int_{E} Q\left(t_{1},(0,0), d\left(z_{1}, w_{1}\right)\right) \int_{E} Q\left(t_{2}-t_{1},\left(z_{1}, w_{1}\right), d\left(z_{2}, w_{2}\right)\right) \int_{E} \\
\cdots \int_{E} Q\left(t_{n}-t_{n-1},\left(z_{n-1}, w_{n-1}\right), d\left(z_{n}, w_{n}\right)\right) \\
\times \chi_{\left\{\left(z_{i}, w_{i}\right)-\left(z_{i-1}, w_{i-1}\right) \in A_{i-1}, i=2, \ldots, n\right\}}\left(\left(z_{1}, w_{1}\right), \ldots,\left(z_{n}, w_{n}\right)\right)
\end{gathered}
$$

which by Lemma 6 is

$$
\begin{aligned}
= & \int_{E} Q\left(t_{1},(0,0), d\left(z_{1}, w_{1}\right)\right) \int_{E} Q\left(t_{2}-t_{1},(0,0), d\left(z_{2}, w_{2}\right)\right) \int_{E} \\
& \cdots \int_{E} Q\left(t_{n}-t_{n-1},(0,0), d\left(z_{n}, w_{n}\right)\right) \chi_{A_{1}}\left(z_{2}, w_{2}\right) \cdots \chi_{A_{n-1}}\left(z_{n}, w_{n}\right) \\
= & Q\left(t_{2}-t_{1},(0,0), A_{1}\right) \cdots Q\left(t_{n}-t_{n-1},(0,0), A_{n-1}\right) \\
= & P_{(0,0)}\left\{y_{t_{2}}-y_{t_{1}} \in A_{1}\right\} \cdots P_{(0,0)}\left\{y_{t_{n}}-y_{t_{n-1}} \in A_{n-1}\right\} . \quad \text { Q.E.D. }
\end{aligned}
$$

Proposition 1. The constant function 1 is a minimal $Q_{t}$-invariant function.

Proof. It is easy to see that 1 is $Q_{t}$-invariant. To show that 1 is also minimal we let $r$ be another $Q_{t}$-invariant function on $(E, \mathscr{B})$ such that $0 \leqslant r \leqslant 1$, we shall prove that $r$ is a constant.

(a) The stochastic process $\left\{r\left(y_{t}\right): t \geqslant 0\right\}$ forms a martingale, i.e. (i) $E_{(0,0)}\left[r\left(y_{t}\right)\right]<\infty$ for all $t \geqslant 0$, and (ii) for any $s, t(0 \leqslant s<t)$,

$$
E_{(0,0)}\left\{r\left(y_{t}\right) \mid r\left(y_{u}\right): u \leqslant s\right\}=r\left(y_{s}\right)
$$

almost surely $\left[P_{(0,0)}\right]$. (i) follows from the boundedness of $r$. To prove (ii), let $\Delta \in \sigma\left\{r\left(y_{u}\right): u \leqslant s\right\} ;$ then

$$
\begin{aligned}
E_{(0,0)}\left[\chi_{\Delta} r\left(y_{t}\right)\right] & =E_{(0,0)}\left[\chi_{\Delta} E_{y_{s}} r\left(y_{t-s}\right)\right] \\
& =E_{(0,0)}\left[\chi_{\Delta} \int_{E} Q\left(t-s, y_{s}, d(z, w)\right) r(z, w)\right] \\
& =E_{(0,0)}\left[\chi_{\Delta} r\left(y_{s}\right)\right]
\end{aligned}
$$

because $r$ is $Q_{t}$-invariant. The above implies that 


$$
E_{(0,0)}\left\{r\left(y_{t}\right) \mid r\left(y_{u}\right): u \leqslant s\right\}=r\left(y_{s}\right) \text { a.s. }\left[P_{(0,0)}\right] .
$$

(b) The martingale convergence theorem implies that

$$
r_{\infty}=\lim _{n \rightarrow \infty} r\left(y_{n}\right)=\lim _{n \rightarrow \infty} r\left[\sum_{i=1}^{n}\left(y_{i}-y_{i-1}\right)\right]
$$

exists almost surely $\left[P_{(0,0)}\right]$. For each number $a$, the event $\left\{r_{\infty}<a\right\}$ is in the tail $\sigma$-field of the random vectors $\left\{z_{n}: n=1,2, \ldots\right\}$, each of which is the partial sum of a sequence of independent identically distributed random vectors $\left\{y_{i}-y_{i-1} ; i=1,2, \ldots\right\}$ (Lemma 8). By the Hewitt-Savage zero one law, the event $\left\{r_{\infty}<a\right\}$ has $P_{(0,0)}$-probability zero or one. Since $a$ is arbitrary, $r_{\infty}=k$ (a constant) with $P_{(0,0)}$-probability one.

(c) $r_{\infty}$ is a closing random variable for the martingale $\left\{r\left(y_{n}\right): n=0,1,2, \ldots\right\}$ because $r$ is bounded. Thus

$$
k=E_{(0,0)}\left\{r_{\infty} \mid r\left(y_{0}\right), r\left(y_{1}\right), \ldots, r\left(y_{n}\right)\right\}=r\left(y_{n}\right) \text { a.s. }\left[P_{(0,0)}\right]
$$

for every $n=0,1,2, \ldots$ Hence $r(y, n)=k$ a.e. $[q(n, 0, d y)]$ and, hence, a.e. with respect to Lebesgue measure on $R$. For any $(x, u) \in E$, let $n>u$ be any integer. Then

$$
r(x, u)=\int_{E} Q(n-u,(x, u), d(y, v)) r(y, v)=\int_{R} q(n-u, x, d y) r(y, n)=k
$$

since $q(n-u, x, d y)$ has a density with respect to Lebesgue measure. Q.E.D.

Lastly, $h$ is minimal because if $g$ is another invariant function such that $0 \leqslant g \leqslant h$, then $g / h$ is $Q_{t}$-invariant and hence $g=c h$ for some constant $c$ by Proposition 1. This finishes the sufficiency part of Theorem 1.

4. Proof of Theorem 2. Theorem 2 is obtained by solving (a) and (b) of Theorem 1 for $h$. Actually we look for positive solutions, since by Lemma 1 the minimal invariant functions $h$ which satisfy $h(0,0)=1$ are positive. Q.E.D.

AcKNowledgement. This paper is based on part of the author's Ph.D. dissertation written at the University of California, Irvine, under the supervision of Professor Donald A. Darling. The author wishes to express his deep gratitude to him. The author also wishes to thank the referee for his very careful reading of the paper and his many useful comments.

\section{REFERENCES}

1. R. M. Blumenthal and R. K. Getoor, Markov processes and potential theory, Academic Press, New York and London, 1968. MR 41 \#9348.

2. D. A. Darling, When is a fixed number of observations optimal?, Proc. Sixth Berkeley Sympos. Math. Statist. and Probability, Vol. IV, 1972, pp. 33-35. 
3. J. L. Doob, Stochastic processes, Wiley, New York; Chapman and Hall, London, 1953. MR $15,445$.

4. J. L. Doob, J. L. Snell and R. E. Williamson, Application of boundary theory to sums of independent random variables, Contributions to Probability and Statistics, Stanford Univ. Press, Stanford, Calif., 1960, pp. 182-197. MR 22 \# 11416.

5. E. B. Dynkin, Markov processes, Fitmatgiz, Moscow, 1963; English transl., Vols. I, II, Grundlehren math. Wiss., Bände 121, 122, Springer-Verlag, Berlin, 1965; Academic Press, New York. MR 33 \# 1886, 1887.

6. T. L. Lai, Space-time processes, parabolic functions and one-dimensional diffusions, Trans. Amer. Math. Soc. 175 (1973), 409-438. MR 48 \# 12656.

7. H. P. McKean, Jr., Stochastic integrals, Academic Press, New York, 1969. MR 40 \#947.

8. P.-A. Meyer, Processus de Markov. La frontière de Martin, Lecture Notes in Math., vol. 77, Springer-Verlag, Berlin and New York, 1968. MR 39 \# 7669.

9. H. Robbins and D. Siegmund, Statistical tests of power one and the integral representation of solutions of certain partial differential equations, Bull. Inst. Math. Acad. Sinica 1 (1973), no. 1, 93-120. MR 47 \#9737.

Department of Mathematics, University of Singapore, Singapore 10, Republic of SINGAPORE 